\title{
Artroplastia total do quadril não cimentada em pacientes com osteoartrose secundária à doença de Legg-Calvé-Perthes em comparação com a osteoartrose primária: Um estudo caso-controle
}

\section{Cementless Total Hip Arthroplasty in Patients with Osteoarthrosis Secondary to Legg-Calvé-Perthes Disease Compared with Primary Osteoarthrosis: A Case-control Study} \author{
Leandro Ejnisman ${ }^{3(D)}$ Helder de Souza Miyahara3 ${ }^{3(D}$ \\ ${ }^{1}$ Disciplina de Ortopedia e Traumatologia, Faculdade de Medicina da \\ Universidade de Santo Amaro, Universidade de Santo Amaro, São \\ Paulo, SP, Brasil \\ 2 Departamento de Ortopedia e Traumatologia, Faculdade de \\ Medicina, Universidade de São Paulo, São Paulo, SP, Brasil \\ ${ }^{3}$ Grupo de Quadril, Instituto de Ortopedia e Traumatologia do \\ Hospital das Clínicas da Faculdade de Medicina da Universidade de \\ São Paulo, São Paulo, SP, Brasil
}

Dennis Sansanovicz ${ }^{1}$ Alberto Tesconi Croci ${ }^{2}{ }^{(0)}$ José Ricardo Negreiros Vicente ${ }^{3(0)}$

\author{
Henrique de Melo Campos Gurgel ${ }^{3}$ (1)
}

Endereço para correspondência Dennis Sansanovicz, MD, MSc, Rua Professor Enéas de Siqueira Neto, 340, Jardim das Imbuias, São Paulo, SP, 04829-300, Brasil (e-mail: dsansanovicz@prof.unisa.br).

Rev Bras Ortop 2022;57(5):843-850.

\section{Resumo \\ Objetivo Realizar uma avaliação clínica, funcional e radiográfica comparativa da artroplastia total do quadril (ATQ) realizada com prótese não cimentada em casos de osteoartrose secundária à doença de Legg-Calvé-Perthes (DLCP) e em casos de osteoartrose primária. \\ Métodos No presente estudo caso-controle, foram revisados os prontuários dos pacientes internados em um hospital universitário entre os anos de 2008 e 2015 . Os \\ Palavras-chave pacientes foram submetidos a ATQ devido a sequelas da DLCP, sendo comparados com \\ - doença de Legg- um grupo controle de pacientes submetidos à mesma cirurgia por osteoartrose Calvé-Perthes \\ - osteoartrose \\ - artroplastia de quadril \\ - complicaçães intraoperatórias primária do quadril. Os pacientes foram recrutados para a realização de uma análise clínica, funcional e radiográfica, na qual foram comparadas as avaliações no pós- operatório imediato e na última consulta de acompanhamento, levando em conside- ração o tempo cirúrgico, o tamanho dos componentes protéticos e as complicações. Resultados Comparamos 22 pacientes do grupo de estudo (25 quadris) com 22 pacientes (25 quadris) do grupo controle, todos os quais foram submetidos a ATQ com}

Trabalho desenvolvido no Instituto de Ortopedia e Traumatologia do Hospital das Clínicas da Faculdade de Medicina da Universidade de São Paulo, São Paulo, SP, Brasil. recebido

04 de Agosto de 2020

aceito

11 de Fevereiro de 2021

Publicado on-line

Outubro 1, 2021
DOI https://doi.org/

10.1055/s-0041-1732330. ISSN 0102-3616. (c) 2021. Sociedade Brasileira de Ortopedia e Traumatologia. All rights reserved.

This is an open access article published by Thieme under the terms of the Creative Commons Attribution-NonDerivative-NonCommercial-License, permitting copying and reproduction so long as the original work is given appropriate credit. Contents may not be used for commercial purposes, or adapted, remixed, transformed or built upon. (https://creativecommons.org/ licenses/by-nc-nd/4.0/)

Thieme Revinter Publicações Ltda., Rua do Matoso 170, Rio de Janeiro, RJ, CEP 20270-135, Brazil 


\author{
Abstract \\ Keywords \\ - Legg-Calvé-Perthes \\ disease \\ - osteoarthrosis \\ - arthroplasty, \\ replacement, hip \\ - intraoperative \\ complications
}

a mesma prótese não cimentada. Houve um maior comprometimento funcional no grupo de pacientes com sequelas da $\operatorname{DLCP}(p=0,002)$. Ocorreram 4 fraturas periprotéticas femorais no intraoperatório do grupo $D L C P$, sendo que não ocorreu nenhuma no grupo de osteoartrose primária $(p=0,050)$.

Conclusões Existe um risco elevado de fratura periprotética femoral no intraoperatório com resultados clínico-funcionais mais desfavoráveis aos pacientes que foram submetidos à ATQ não cimentada devido a osteoartrose secundária às sequelas da DLCP do que naqueles que foram submetidos à mesma cirurgia por osteoartrose primária de quadril.

Objective To perform a comparative clinical, functional and radiographic evaluation of total hip arthroplasty (THA) performed with a cementless prosthesis in cases of osteoarthrosis secondary to Legg-Calvé-Perthes Disease (LCPD) and in cases of primary osteoarthrosis.

Methods In the present case-control study, we reviewed medical records of patients admitted to a university hospital between 2008 and 2015 to undergo THA due to LCPD sequelae and compared them with a control group of patients who underwent the same surgery due to primary hip osteoarthrosis. We recruited patients for clinical, functional, and radiographic analysis and we compared the evaluations in the immediate postoperative period and at the last follow-up visit, considering surgical time, size of prosthetic components, and complications.

Results We compared 22 patients in the study group ( 25 hips) with 22 patients ( 25 hips) in the control group, all of whom had undergone THA with the same cementless prosthesis. There was greater functional impairment in the group of patients with LCPD sequelae $(p=0.002)$. There were 4 intraoperative femoral periprosthetic fractures in the LCPD group and none in the primary osteoarthrosis group $(p=0.050)$.

Conclusions There is an increased risk of intraoperative periprosthetic femoral fracture and worse clinical-functional results in patients undergoing cementless THA due to osteoarthrosis secondary to LCPD sequelae than in those who have undergone the same surgery due to primary hip osteoarthrosis.

\section{Introdução}

Entre todas as artroplastias totais do quadril (ATQs) devido a osteoartrose do quadril, 0,6 a 4,2\%, são de casos secundários às sequelas da doença de Legg-Calvé-Perthes (DLCP). ${ }^{1-6}$ Até 0 momento do presente estudo, apenas oito séries de casos, um estudo de caso-controle e uma revisão sistemática foram publicados sobre ATQ com a finalidade de tratar as sequelas da DLCP, sendo que em apenas oito desses estudos foram descritas complicações do intra e do pós-operatório. ${ }^{4,7-15}$ No entanto, as deformidades típicas do fêmur proximal e do acetábulo em pacientes com DLCP tornam a ATQ um desafio para o cirurgião do quadril. ${ }^{1,6,7}$

Aproximadamente entre 3 e $6 \%$ dos pacientes com sequelas da DLCP submetidos a ATQ podem vir a apresentar déficit neurológico - uma taxa consideravelmente mais elevada do que o risco geral de $0,17 \%$, de déficit neurológico após ATQ realizada por qualquer outro motivo. ${ }^{4,16} \mathrm{~A}$ desigualdade no comprimento dos membros após a cirurgia é outra possível complicação entre os pacientes com DLCP, ${ }^{17}$ assim como o risco de fratura femoral intraoperatória., ${ }^{4,15}$

No entanto, a literatura é pobre em estudos sobre ATQ que comparam pacientes submetidos a cirurgia para osteoar- trose secundária às sequelas da DLCP com aqueles pacientes que apresentam outras doenças degenerativas do quadril. Presumimos que a ATQ realizada por sequelas da DLCP, devido às suas dificuldades técnicas, pode estar associada a um risco mais elevado de complicações perioperatórias e a desfechos clínicos e funcionais mais desfavoráveis do que em pacientes submetidos a cirurgia para osteoartrose primária do quadril.

O objetivo principal do presente estudo foi realizar uma avaliação clínica, funcional e radiográfica comparativa da ATQ realizada com um modelo de prótese não cimentada em casos de osteoartrose secundária à DLCP e em casos de osteoartrose primária. O objetivo secundário foi comparar os dois grupos quanto às complicações.

\section{Materiais e Métodos}

\section{Desenho do Estudo e Ética}

Trata-se de um estudo caso-controle baseado na revisão dos prontuários e na avaliação funcional e clínica dos pacientes submetidos em um hospital universitário a ATQ devido a osteoartrose do quadril. Foram comparados os pacientes submetidos a ATQ por osteoartrose secundária às sequelas 
da DLCP com os pacientes submetidos à mesma cirurgia por osteoartrose primária do quadril.

O conselho de ética local aprovou o protocolo do estudo. Os pacientes ou seus responsáveis legais assinaram o termo de consentimento informado para fins de participação no estudo e do uso das imagens radiográficas na presente publicação.

\section{Participantes e Grupos}

Foram revisados os prontuários de todos os pacientes internados para ATQ entre os anos de 2008 e 2015. Para fins de padronização e evitar viés na avaliação dos resultados clínicos, selecionamos apenas os pacientes submetidos a cirurgia no nosso hospital nos quais foi utilizado um modelo específico de prótese. Foram excluídos os pacientes operados com outros modelos ou materiais de próteses do quadril. Este modelo (prótese não cimentada Groupe Lépine, fabricado em Genay, França) possui um componente femoral de liga de titânio com superfície porosa revestida com hidroxiapatita (Targos, Group Lepine, fabricado em Genay, França) e um componente acetabular feito de liga de titânio com superfície porosa e revestida com hidroxiapatita (modelo MBA). A cabeça protética é feita de aço inoxidável ou de cerâmica de alumina, com $28 \mathrm{~mm}$ de diâmetro, e o revestimento/inserto é composto de polietileno.

Os pacientes submetidos a ATQ devido a osteoartrite secundária a outras doenças que não foram causadas por sequelas da DLCP foram excluídos. Também foram excluídos os pacientes sem identificação da causa da osteoartrose e aqueles sem acompanhamento mínimo de 2 anos. No entanto, não foram excluídos os pacientes com osteoartrose primária.

Assim que identificamos todos os pacientes submetidos a ATQ por sequelas da DLCP, examinamos cuidadosamente os prontuários em busca de confirmação de que a doença havia sido diagnosticada na infância (com radiografias realizadas no início da instituição, com fise aberta) e convidamos esses pacientes a vir ao hospital para a realização de uma avaliação clínica. Os pacientes cujos diagnósticos nossa equipe não conseguiu confirmar que datavam da infância foram excluídos.

Criamos, então, um grupo de pacientes submetidos a ATQ devido a osteoartrose primária no mesmo período, pareados com o grupo de estudo de pacientes (1:1) com sequelas da DLCP, os quais foram separados por gênero, lateralidade e tempo de acompanhamento. Em ambos os grupos, todos os pacientes foram submetidos a ATQ pelo emprego da abordagem lateral direta de Hardinge.

\section{Resultados Clínicos e Demográficos}

Foram examinados os prontuários médicos com o objetivo de coletar dados demográficos e clínicos, incluindo o tempo cirúrgico, o tamanho dos componentes protéticos, as complicações intra- e pós-operatórias e qualquer indicação de revisão cirúrgica. Os resultados clínicos foram avaliados por meio do questionário Lequesne. ${ }^{18}$

\section{Resultados Radiográficos}

Foram avaliadas as radiografias nas incidências anteroposteriores do pós-operatório imediato e do último acompanhamento. Todos estes exames digitais foram avaliados com o auxílio do software Philips DICOM Viewer R3.0-SP03 (Koninklijke Philips N.V., Eindhoven, Holanda) a fim de que pudéssemos calcular a inclinação do componente acetabular em relação à pelve, o deslocamento femoral, pelo método de Sundsvall, ${ }^{19}$ e a posição do componente femoral em relação ao canal femoral.

O software calculou automaticamente a inclinação do componente acetabular em relação à pelve usando o ângulo entre as duas seguintes linhas na radiografia: uma linha unindo a borda proximal e a lateral, com a borda distal e medial da maior circunferência do componente acetabular, e a outra linha unindo as regiões mais distais das duas tuberosidades isquiáticas ( - Fig. 1). Em seguida, calculamos o deslocamento femoral lateral por meio do método de Sundsvall (- Fig. 2). ${ }^{19}$

Foi avaliada também a posição do componente femoral em relação ao canal femoral (centralização). Foi usado o mesmo software para medir (em milímetros) a distância entre a região mais distal do componente da prótese femoral e a extremidade interna adjacente da cortical femoral medial e a distância entre a região mais distal do componente
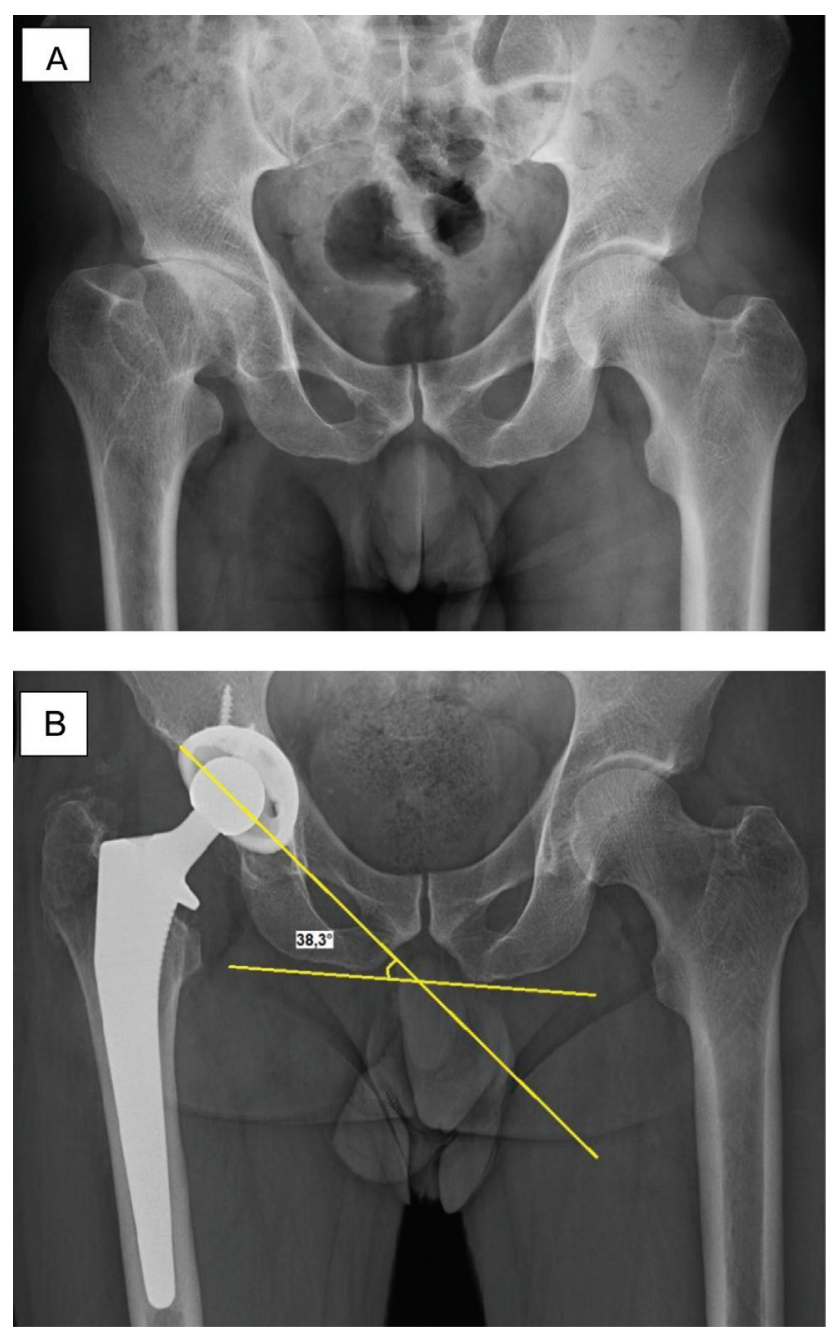

Fig. 1 Inclinação do componente acetabular em relação à pelve. Demonstração das linhas usadas para calcular o ângulo nas radiografias do quadril em incidência anteroposterior. (A) Incidência radiográfica pré-operatória. (B) Acompanhamento final e cálculo do ângulo. 

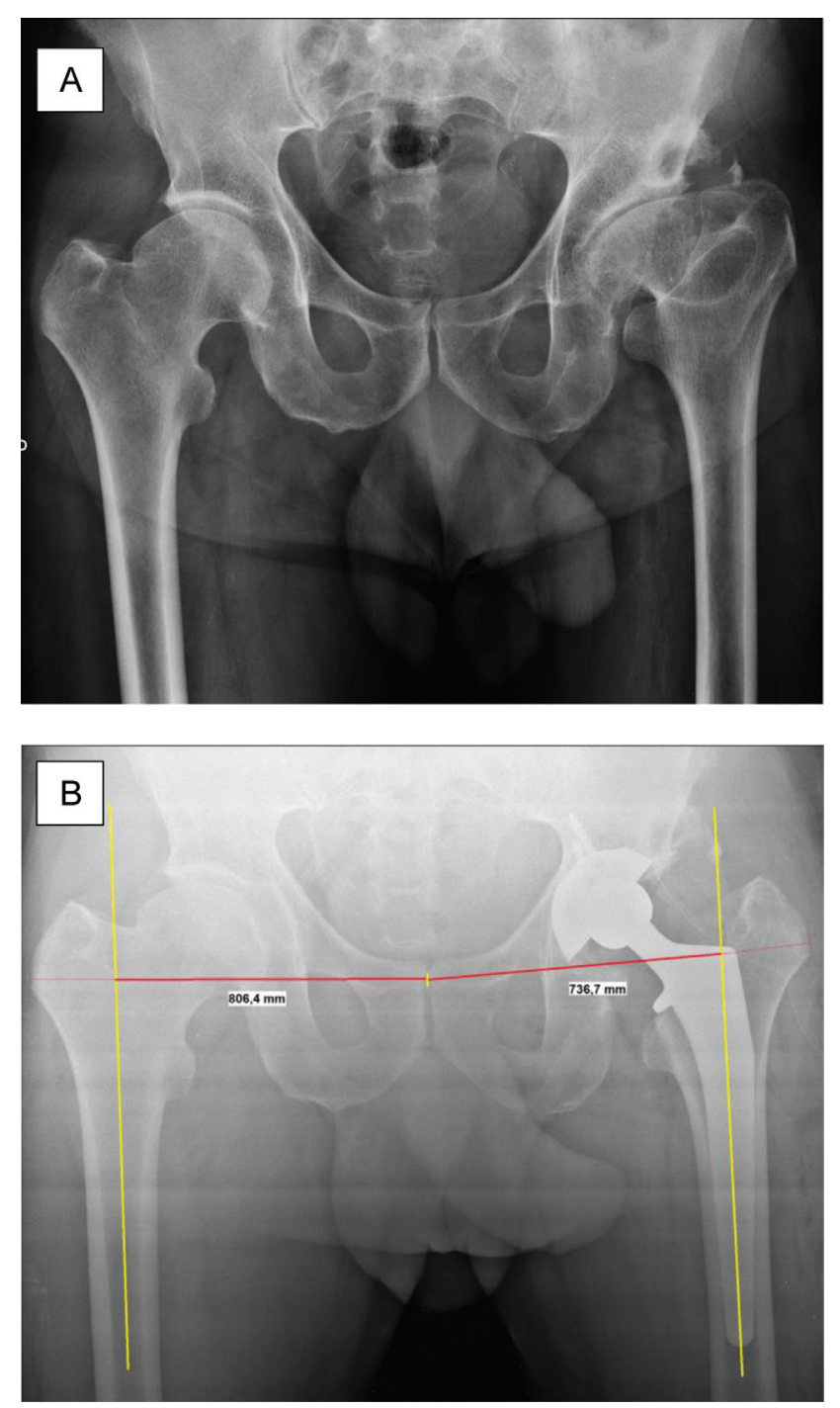

Fig. 2 Cálculo do deslocamento femoral em incidências radiográficas anteroposteriores do quadril. (A) Período pré-operatório. (B) Período pós-operatório imediato, com deslocamento femoral lateral diferencial de $-69,7 \mathrm{~mm}$.

femoral e a extremidade interna adjacente da cortical lateral do componente. Calculamos uma divisão entre as duas distâncias, considerando que o resultado é próximo de 1 quando os componentes femorais estão centralizados no canal femoral, $<1$ quando estão "em valgo", e $>1$ quando estão "em varo" (- Fig. 3).

$O$ pesquisador principal fez todas as medidas nas radiografias, as quais foram comparadas com todas as medidas entre os grupos de estudo.

\section{Análise Estatística}

Os dados foram registrados em planilhas do Microsoft Excel (Microsoft Corporation, Redmond, WA, EUA) e transferidos para o software IBM SPSS Statistics for Mac, versão 23.0 (IBM Corp., Armonk, NY, EUA) para fins de análise estatística. Comparamos os dados categóricos entre os grupos utilizando o teste de qui-quadrado de Pearson. Empregou-se o teste de Kolmogorov-Smirnov para verificar a normalidade da distribuição dos dados para variáveis contínuas. Em seguida, usa-
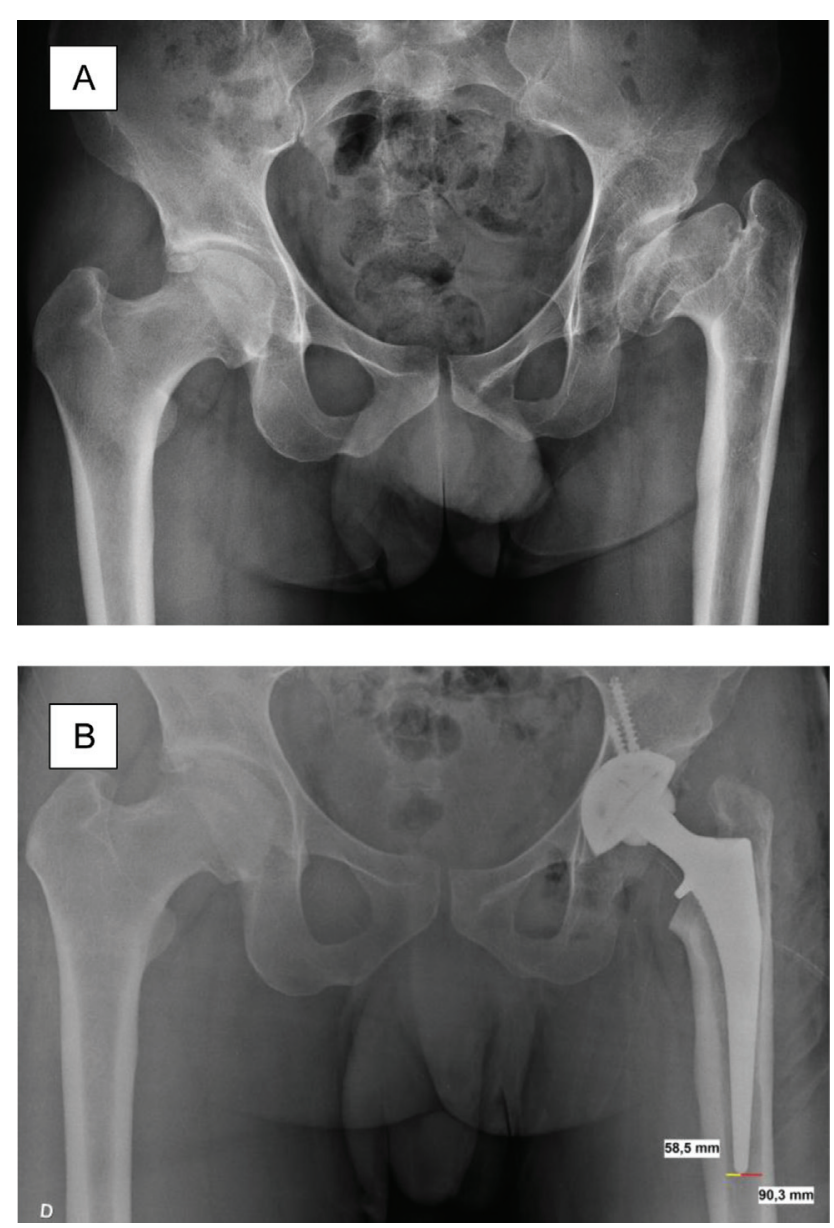

Fig. 3 Avaliação da posição do componente femoral em relação ao canal femoral (centralização) nas incidências radiográficas anteroposteriores do quadril. (A) Período pré-operatório. (B) Período pósoperatório imediato.

mos o teste $t$ de Student para os dados distribuídos normalmente em amostras independentes ou o teste não paramétrico de Mann-Whitney para os dados não gaussianos (como para o escore funcional de Lequesne). Foi aceito um erro tipo $\mathrm{I} \leq 5 \%$ como diferença estatisticamente significativa.

\section{Resultados}

\section{Grupos do Estudo e Pareamento}

Durante o período do presente estudo, 810 pacientes foram submetidos a ATQ em nossa instituição, utilizando o modelo padronizado para o presente estudo. Todos foram submetidos à cirurgia mediante abordagem lateral direta do quadril. Identificamos 144 pacientes com osteoartrose primária, e 49 foram operados por sequelas da DLCP (6\%). Após uma revisão dos prontuários e do comparecimento para as consultas clínicas, foram excluídos pacientes deste grupo pelos motivos descritos no fluxograma da - Fig. 4. Também foram excluídos 93 pacientes com idade $>60$ anos do grupo controle. Tanto o grupo DLCP final quanto o grupo controle final, com pacientes com osteoartrose primária, foram formados por 22 pacientes (25 quadris). A - Tabela 1 mostra que os grupos foram homogêneos quanto ao gênero, aos lados em que foram submetidos à cirurgia, e ao tempo de acompanhamento. 


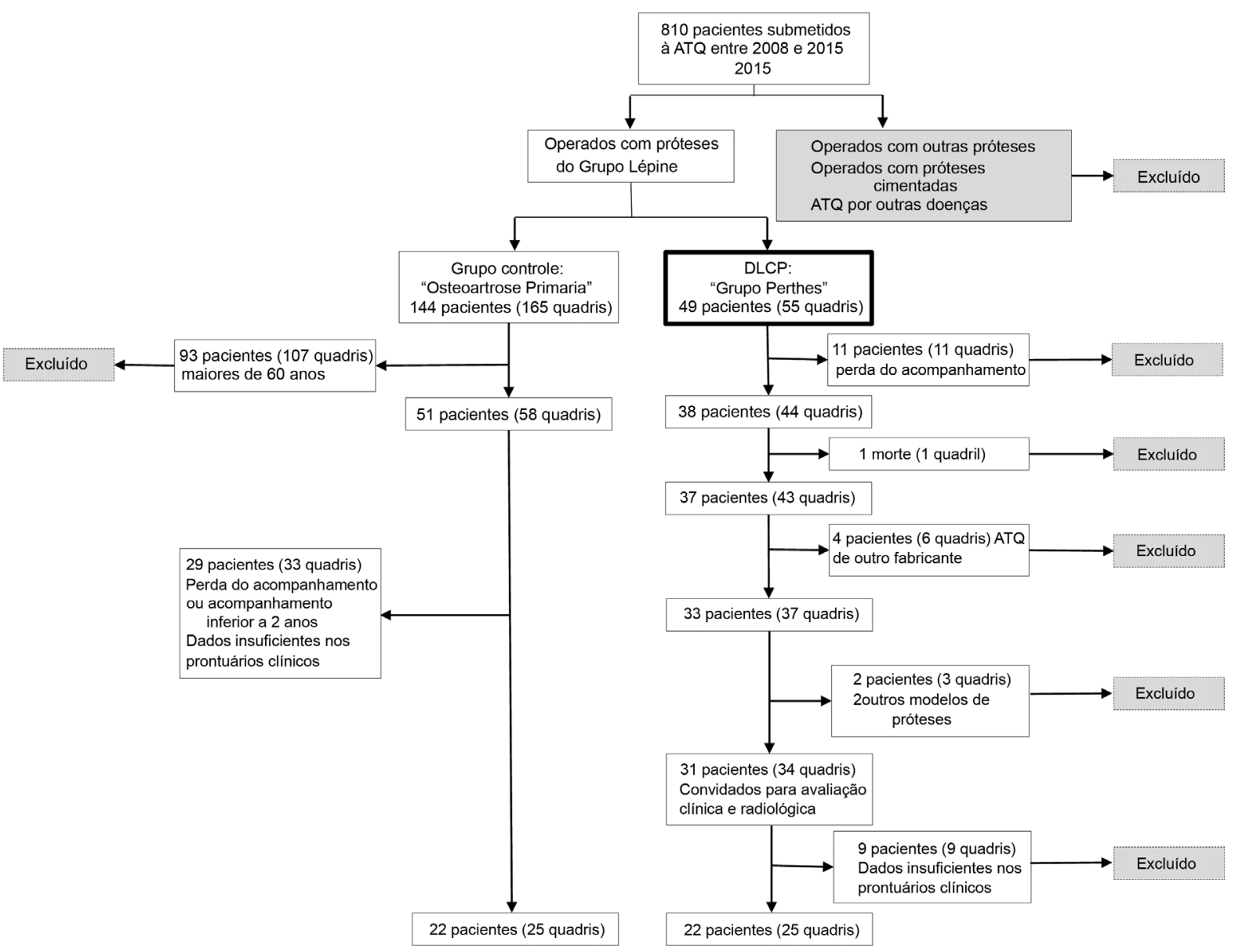

Fig. 4 Fluxograma de inclusão e exclusão de pacientes no estudo em ambos os grupos.

Tabela 1 Frequências absolutas e relativas de gênero, idade, lado operado e tempo de acompanhamento no grupo com doença de Legg-Calvé-Perthes (DLCP) e no grupo controle dos pacientes com osteoartrose primária do quadril

\begin{tabular}{|c|c|c|c|}
\hline & $\begin{array}{l}\text { Grupo DLCP } \\
(n=25 \text { quadris) }\end{array}$ & $\begin{array}{l}\text { Grupo controle } \\
(n=25 \text { quadris })\end{array}$ & valor-p \\
\hline \multicolumn{4}{|l|}{ Gênero, n (\%) } \\
\hline Masculino & $17(68)$ & $12(48)$ & \multirow[t]{2}{*}{0,152} \\
\hline Feminino & $8(32)$ & $13(52)$ & \\
\hline \multicolumn{4}{|l|}{ Idade, anos } \\
\hline Média (DP) & $47,3(7,4)$ & $53,2(4,4)$ & \multirow[t]{2}{*}{0,001} \\
\hline Mediana (IQR) & $46,0(42,0-53,0)$ & $54,0(50,0-56,0)$ & \\
\hline \multicolumn{4}{|c|}{ Lado operado, n (\%) } \\
\hline Direito & $12(48)$ & $12(48)$ & \multirow[t]{3}{*}{$>0,999$} \\
\hline Esquerdo & $13(52)$ & $13(52)$ & \\
\hline Bilateral & $3(12)$ & $3(12)$ & \\
\hline \multicolumn{4}{|c|}{ Tempo de acompanhamento, meses } \\
\hline Média (DP) & $62.2(18,9)$ & $65,3(15,3)$ & \multirow[t]{2}{*}{0,052} \\
\hline Mediana (IQR) & $59,0(52,0-67,0)$ & $62,0(57,0-73,0)$ & \\
\hline
\end{tabular}

Abreviações: DLCP, doença de Legg-Calvé-Perthes; DP, desvio padrão; IQR, intervalo interquartil.

\section{Desfechos Cirúrgicos}

O tempo cirúrgico $(p=0,62)$ e o tamanho dos componentes da prótese femoral $(p=0,174)$ e acetabular $(p=0,149)$ foram semelhantes entre os grupos. No grupo DLCP, ocorreram quatro fraturas periprotéticas durante a cirurgia na região da metáfise femoral e uma fratura de avulsão do trocanter maior. Todas as fraturas metafisárias do fêmur foram tratadas no mesmo procedimento, com fios de cerclagem. A 


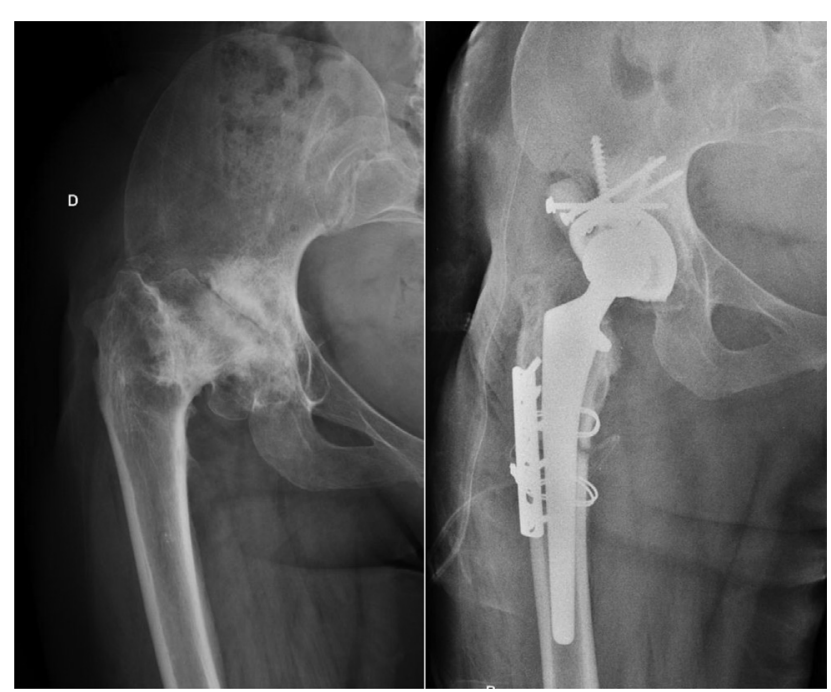

Fig. 5 Incidências radiográficas anteroposteriores pré-operatórias (esquerda) e pós-operatórias imediatas (direita), evidenciando enxerto ósseo impactado no teto acetabular e osteotomia de encurtamento na região subtrocantérica.

fratura de avulsão do trocanter maior não foi descrita no prontuário clínico, mas foi observada na radiografia pósoperatória imediata. No grupo Osteoartrose Primária, não houve registros ou imagens radiográficas que demonstrassem fraturas periprotéticas intraoperatórias. A diferença entre os grupos quanto à frequência de fraturas foi estatisticamente significativa $(p=0,050)$.

No grupo DLCP, foi necessário em quatro casos o uso de enxerto ósseo autólogo (da cabeça ou colo do fêmur) para a fixação e um melhor posicionamento do componente acetabular. Foram 3 enxertos estruturais no teto acetabular, fixados com parafusos corticais $(4,5 \mathrm{~mm})$ e 1 enxerto impactado na região medial do acetábulo, a fim de preencher a falha do componente medial. Nenhum paciente do grupo controle necessitou de enxerto ósseo $(p=0,109)$. Em um paciente do Grupo DLCP, foi necessária osteotomia de encurtamento femoral na região subtrocantérica, sendo fixada com placa, parafusos e fios de cerclagem (-Fig. 5).

\section{Resultados Clínicos e Funcionais}

A pontuação média na avaliação de Lequesne para os pacientes do grupo DLCP foi 9,1 $\pm 4,7$, indicando comprometimento funcional grave, e 4,8 \pm 4,0 para o grupo controle (diferença média de 4,3 pontos; intervalo de confiança [IC] de 95\%: 1,8$6,7 ; p=0,002)$. A distribuição dos pacientes segundo as categorias dos escores funcionais é apresentada na - Tabela 2. Em nenhum dos casos entre os grupos estudados houve episódios de infecção, de luxação ou de dano neurológico em decorrência da cirurgia. Nenhuma cirurgia de revisão foi indicada ou realizada em nenhum grupo.

\section{Resultados Radiográficos}

Nas radiografias pós-operatórias imediatas, a inclinação média do componente acetabular em relação à pelve foi de $44,1 \pm 6,4^{\circ}$ para o grupo DLCP e de $43,8 \pm 6,2^{\circ}$ para o grupo controle, com diferença média entre os grupos de $0,3^{\circ}$ (IC95\%: - 3,9-3,26;
Tabela 2 Distribuição dos pacientes segundo categorias de escores funcionais de Lequesne

\begin{tabular}{|c|c|c|c|}
\hline Categorias de deficiência & $\begin{array}{l}\text { Grupo } \\
\text { DLCP }\end{array}$ & $\begin{array}{l}\text { Grupo } \\
\text { controle }\end{array}$ & valor-p \\
\hline Leve (1 a 4), n (\%) & $4(16)$ & $17(68)$ & \multirow[t]{5}{*}{0,002} \\
\hline Moderada (5 a 7), n (\%) & $6(24)$ & $4(16)$ & \\
\hline Grave (8 a 10), n (\%) & $5(20)$ & $2(8)$ & \\
\hline Muito grave (11 a 13), n (\%) & $5(20)$ & $1(4)$ & \\
\hline Extremamente grave ( $\geq 14), \mathrm{n}(\%)$ & $5(20)$ & $1(4)$ & \\
\hline
\end{tabular}

Abreviação: DLCP, Doença de Legg-Calvé-Perthes.

Tabela 3 Posição do componente femoral em relação ao canal femoral (centralização): distância média em milímetros

\begin{tabular}{|l|l|l|l|l|l|l|l|}
\hline & \multicolumn{3}{|l|}{ Grupo DLCP } & \multicolumn{4}{|c|}{ Grupo controle } \\
\cline { 2 - 8 } & $\boldsymbol{n}$ & Média & DP & $\boldsymbol{n}$ & Média & DP & valor-p \\
\hline $\begin{array}{l}\text { Período pós-opera- } \\
\text { tório imediato }\end{array}$ & 25 & 0,9 & 0,2 & 25 & 1,3 & 0,6 & 0,008 \\
\hline $\begin{array}{l}\text { Último } \\
\text { acompanhamento }\end{array}$ & 25 & 0,9 & 0,4 & 25 & 1,4 & 0,7 & 0,002 \\
\hline
\end{tabular}

Abreviações: DLCP, Doença de Legg-Calvé-Perthes; DP, desvio padrão.

$p=0,628)$. No último acompanhamento, os valores médios foram de $43,2 \pm 6,9^{\circ}$ e de $43,0^{\circ} \pm 6,2^{\circ}$, respectivamente, com diferença média entre os grupos de $0,2^{\circ}$ (IC95\%: - 3,9-3,6; $p=0,497)$. O deslocamento femoral médio foi semelhante entre os grupos ( $p=0,079$ para o pós-operatório imediato e $p=0,273$ para o último acompanhamento).

A - Tabela 3 mostra os resultados para a posição do componente femoral em relação ao canal femoral. A "centralização" foi significativamente diferente entre os grupos (diferença média de - 0,4; IC95\%: - 0,1-- 0,7 no pós-operatório imediato; e de -0,5; IC95\%: - 0,2-- 0,8 no último acompanhamento). Em ambos os momentos da avaliação, os componentes femorais da prótese tendem a ser implantados mais em valgo no canal femoral (relação $<1,0$ ) no grupo DLCP do que nos pacientes com osteoartrose primária do quadril (relação $>1,0$ ).

\section{Discussão}

Até onde sabemos, o presente estudo é o primeiro na literatura a comparar os resultados clínicos, funcionais e radiográficos das cirurgias de ATQ. As cirurgias foram realizadas em pacientes com DLCP e com osteoartrose primária, sendo empregado na cirurgia o mesmo modelo de prótese não cimentada. Tivemos o cuidado de excluir os pacientes que foram submetidos a ATQ com outros tipos e modelos de componentes protéticos a fim de evitar a interferência de fatores de confusão nos desfechos clínicos. Observamos que, mesmo com o uso do mesmo produto, os pacientes com sequelas de DLCP apresentam maior risco de fraturas periprotéticas, apresentando resultados clínico-funcionais mais desfavoráveis do que os pacientes com osteoartrose primária do quadril. Estes achados evidenciam o desafio técnico da 
realização da ATQ imposta pelas deformidades da DLCP, exigindo tempos cirúrgicos de $142,4^{3}$ a $154,8^{13}$ minutos, o que demonstra a necessidade de se realizar mais estudos clínicos com a finalidade de abordar essas questões.

A cavidade acetabular nos pacientes com osteoartrose secundária às sequelas de DLCP está deformada morfologicamente, tornando-se rasa, com diâmetro aumentado e retrovertida em relação à pelve. ${ }^{6,20}$ Esta alteração estrutural pode dificultar o implante dos componentes acetabulares convencionais. No nosso estudo, embora os diâmetros médios dos componentes acetabulares implantados tenham sido semelhantes nos grupos DLCP e controle estudados, foi necessário o uso de enxerto ósseo autólogo em quatro casos do grupo DLCP para o correto posicionamento dos componentes. Estes dados sugerem que a deformidade da cavidade acetabular na osteoartrose secundária às sequelas da DLCP acarreta dificuldades técnicas para o implante dos componentes acetabulares convencionais.

Embora a taxa de fratura femoral periprotética intraoperatória seja em torno de $3 \%$ na ATQ não cimentada por qualquer motivo, ${ }^{13}$ a taxa desta complicação pode ser muito maior em pacientes com sequelas da DLCP, chegando a $13,8 \%$ 15 com o uso de componentes convencionais. Al-Khateeb et al. ${ }^{9}$ personalizaram o componente femoral de acordo com as imagens tomográficas pré-operatórias e não relataram nenhuma fratura intraoperatória. Seufert et al. ${ }^{11}$ usaram componentes curtos modulares na ATQ em uma tentativa de superar a anatomia anormal dos pacientes com DLCP. Estes autores também não relataram nenhuma fratura. Avaliamos os pacientes operados com componentes femorais convencionais e o percentual desta complicação atingiu $20 \%$. Na osteoartrose secundária às sequelas da DLCP, as deformidades femorais não se limitam ao sítio da necrose na infância; ou seja, além das deformidades descritas na cabeça femoral, ${ }^{6}$ o colo femoral é encurtado em relação à extensão e à medialização do trocanter maior. ${ }^{21,22} 0$ ângulo cervicodiafisário diminui, produzindo uma deformidade em varo, existindo uma incongruência morfológica entre a metáfise e a diáfise femoral. ${ }^{22,23}$ Acreditamos que, dependendo da gravidade da deformidade femoral, o uso dos componentes femorais modulares ou personalizados deve ser considerado, com o objetivo de minimizar o risco de fratura femoral periprotética intraoperatória.

A deformação morfológica do quadril nos pacientes com DLCP, além de dificultar a técnica cirúrgica e gerar mais complicações, também pode afetar os resultados clínicofuncionais da ATQ. ${ }^{6,24-28}$ Nossos pacientes com DLCP apresentaram maior comprometimento funcional no último acompanhamento. do que os pacientes submetidos a cirurgia de osteoartrose primária do quadril.

A inclinação média do componente acetabular no presente estudo $\left(44,1^{\circ}\right.$ no período pós-operatório imediato e $43,2^{\circ}$ no acompanhamento tardio) foi semelhante aos números obtidos em outros estudos com pacientes com DLCP, ${ }^{7,11-14}$ e permaneceu dentro da faixa proposta por Lewinnek et al. ${ }^{29}$ como segura $\left(30\right.$ a $50^{\circ}$ ). 0 presente estudo foi o primeiro a medir o deslocamento femoral após uma ATQ para sequelas da DLCP. Portanto, não há referências prévias para esta medida em pacientes com deformidades acometidos pela DLCP, sendo necessários mais estudos para verificar se os valores encontrados explicam os resultados da ATQ nesses pacientes.

Verificamos que os componentes femorais da prótese tendem a ser implantados mais em valgo no canal femoral na DLCP do que nos casos de osteoartrose primária do quadril. Este achado difere dos resultados de Traina et al. ${ }^{7}$ e de Pietrzak et al., ${ }^{8}$ que consideraram esta posição mais neutra. No entanto, o método de cálculo desta característica não estava bem descrito nesses estudos, dificultando as comparações.

O nosso estudo apresenta limitações. Não foi possível controlar ou verificar a posição do paciente na mesa durante o exame radiográfico, devido à natureza retrospectiva do presente estudo. Porém, todas as radiografias foram realizadas na mesma instituição, seguindo os mesmos protocolos. Outra característica impossível de controlar no presente estudo foi a técnica cirúrgica, já que diferentes cirurgiões realizaram a ATQ nesta série de casos. Como os pacientes foram submetidos a cirurgia em um hospital universitário, a curva de aprendizado também pode impactar os resultados cirúrgicos.

\section{Conclusões}

Os pacientes submetidos à ATQ não cimentada devido à osteoartrite secundária às sequelas de DLCP apresentam risco elevado de fratura periprotética do fêmur no intraoperatório, apresentando resultados clínico-funcionais mais desfavoráveis do que os pacientes que foram submetidos à mesma cirurgia devido a osteoartrose primária do quadril.

\section{Suporte Financeiro}

O presente estudo não recebeu financiamento ou apoio financeiro de qualquer espécie.

\section{Conflito de Interesses}

Os autores declaram não haver conflito de interesses.

\section{Referências}

1 Thillemann TM, Pedersen AB, Johnsen SP, Søballe KDanish Hip Arthroplasty Registry. Implant survival after primary total hip arthroplasty due to childhood hip disorders: results from the Danish Hip Arthroplasty Registry. Acta Orthop 2008;79(06): 769-776

2 Furnes O, Lie SA, Espehaug B, Vollset SE, Engesaeter LB, Havelin LI. Hip disease and the prognosis of total hip replacements. A review of 53,698 primary total hip replacements reported to the Norwegian Arthroplasty Register 1987-99. J Bone Joint Surg Br 2001;83 (04):579-586

3 Boyd HS, Ulrich SD, Seyler TM, Marulanda GA, Mont MA. Resurfacing for Perthes disease: an alternative to standard hip arthroplasty. Clin Orthop Relat Res 2007;465(465):80-85

4 Baghdadi YMK, Larson AN, Stans AA, Mabry TM. Total hip arthroplasty for the sequelae of Legg-Calvé-Perthes disease. Clin Orthop Relat Res 2013;471(09):2980-2986

5 Uluçay C, Ozler T, Güven M, Akman B, Kocadal AO, Altıntaş F Etiology of coxarthrosis in patients with total hip replacement. Acta Orthop Traumatol Turc 2013;47(05):330-333 
6 Gent E, Clarke NMP. Joint replacement for sequelae of childhood hip disorders. J Pediatr Orthop 2004;24(02):235-240

7 Traina F, De Fine M, Sudanese A, Calderoni PP, Tassinari E, Toni A. Long-term results of total hip replacement in patients with LeggCalvé-Perthes disease. J Bone Joint Surg Am 2011;93(07):e25

8 Pietrzak K, Strzyzewski W, Pucher A, Kaczmarek W. [Total hip replacement after Legg-Calvé-Perthes disease]. Pol Orthop Traumatol 2011;76(03):129-133

9 Al-Khateeb H, Kwok IHY, Hanna SA, Sewell MD, Hashemi-Nejad A. Custom cementless THA in patients with Legg-Calve-Perthes Disease. J Arthroplasty 2014;29(04):792-796

10 Lim YW, Kim MJ, Lee YS, Kim YS. Total Hip Arthroplasty in Patient with the Sequelae of Legg-Calvé-Perthes Disease. Hip Pelvis 2014; 26(04):214-219

11 Seufert CR, McGrory BJ. Treatment of Arthritis Associated With Legg-Calve-Perthes Disease With Modular Total Hip Arthroplasty. J Arthroplasty 2015;30(10):1743-1746

12 Lee SJ, Yoo JJ, Kim HJ. Alumina-alumina total hip arthroplasty for the sequelae of Legg-Calve-Perthes disease: A comparative study with adult-onset osteonecrosis. J Orthop Sci 2016;21(06):836-840

13 Lee KH, Jo WL, Ha YC, Lee YK, Goodman SB, Koo KH. Total hip arthroplasty using a monobloc cementless femoral stem for patients with childhood Perthes' disease. Bone Joint J 2017;99$\mathrm{B}(04): 440-444$

14 Luo ZY, Wang HY, Wang D, Pan H, Pei FX, Zhou ZK. Monobloc implants in cementless total hip arthroplasty in patients with Legg-Calve-Perthes disease: a long-term follow-up. BMC Musculoskelet Disord 2017;18(01):386

15 Hanna SA, Sarraf KM, Ramachandran M, Achan P. Systematic review of the outcome of total hip arthroplasty in patients with sequelae of Legg-Calvé-Perthes disease. Arch Orthop Trauma Surg 2017;137(08):1149-1154

16 Farrell CM, Springer BD, Haidukewych GJ, Morrey BF. Motor nerve palsy following primary total hip arthroplasty. J Bone Joint Surg Am 2005;87(12):2619-2625

17 Krych AJ, Howard JL, Trousdale RT, Cabanela ME, Berry DJ. Total hip arthroplasty with shortening subtrochanteric osteotomy in Crowe type-IV developmental dysplasia: surgical technique. J Bone Joint Surg Am 2010;92(Suppl 1 Pt 2):176-187

18 Marx FC, de Oliveira LM, Bellini CG, Ribeiro MCC. Tradução e validação cultural do questionário algofuncional de Lequesne para osteoartrite de joelhos e quadris para a língua portuguesa. Rev Bras Reumatol 2006;46(04):253-260

19 Kjellberg M, Englund E, Sayed-Noor AS. A new radiographic method of measuring femoral offset. The Sundsvall method. Hip Int 2009;19(04):377-381

20 Sankar WN, Flynn JM. The development of acetabular retroversion in children with Legg-Calvé-Perthes disease. J Pediatr Orthop 2008;28(04):440-443

21 Stulberg SD, Cooperman DR, Wallensten R. The natural history of Legg-Calvé-Perthes disease. J Bone Joint Surg Am 1981;63(07): 1095-1108

22 Froberg L, Christensen F, Pedersen NW, Overgaard S. Radiographic changes in the hip joint in children suffering from Perthes disease. J Pediatr Orthop B 2012;21(03):220-225

23 Kitakoji T, Hattori T, Kitoh H, Katoh M, Ishiguro N. Which is a better method for Perthes' disease: femoral varus or Salter osteotomy? Clin Orthop Relat Res 2005;(430):163-170

24 Sanchez-Sotelo J, Berry DJ, Trousdale RT, Cabanela ME. Surgical treatment of developmental dysplasia of the hip in adults: II. Arthroplasty options. J Am Acad Orthop Surg 2002;10(05): 334-344

25 Zhu J, Wang Y, Pang J, et al. [Effectiveness of total hip arthroplasty for severe developmental dysplasia of hip in adults]. Zhongguo Xiu Fu Chong Jian Wai Ke Za Zhi 2014;28(03): 335-338

26 Kohlhof H, Ziebarth K, Gravius S, Wirtz DC, Siebenrock KA. Die operative Versorgung der sekundären Coxarthrose bei kongenitaler Hüftluxation (Crowe Typ IV). Oper Orthop Traumatol 2013; 25(05):469-482

27 Larson AN, McIntosh AL, Trousdale RT, Lewallen DG. Avascular necrosis most common indication for hip arthroplasty in patients with slipped capital femoral epiphysis. J Pediatr Orthop 2010;30 (08):767-773

28 Schoof B, Citak M, O'Loughlin PF, et al. Eleven year results of total hip arthroplasty in patients with secondary osteoarthritis due to slipped capital femoral epiphysis. Open Orthop J 2013;7(01): 158-162

29 Lewinnek GE, Lewis JL, Tarr R, Compere CL, Zimmerman JR. Dislocations after total hip-replacement arthroplasties. J Bone Joint Surg Am 1978;60(02):217-220 